Research Article

\title{
The Conical Radial Basis Function for Partial Differential Equations
}

\author{
J. Zhang, ${ }^{1}$ F. Z. Wang $\mathbb{D}^{1,2,3}$ and E. R. Hou $\mathbb{D}^{1}$ \\ ${ }^{1}$ College of Computer Science and Technology, Huaibei Normal University, Huaibei 235000, China \\ ${ }^{2}$ Nanchang Institute of Technology, Nanchang 330044, China \\ ${ }^{3}$ School of Mathematical and Statistics, Xuzhou University of Technology, Xuzhou 221018, Jiangsu, China
}

Correspondence should be addressed to F. Z. Wang; wangfuzhang1984@163.com

Received 19 October 2020; Revised 25 October 2020; Accepted 29 October 2020; Published 11 November 2020

Academic Editor: Imtiaz Ahmad

Copyright $\odot 2020 \mathrm{~J}$. Zhang et al. This is an open access article distributed under the Creative Commons Attribution License, which permits unrestricted use, distribution, and reproduction in any medium, provided the original work is properly cited.

\begin{abstract}
The performance of the parameter-free conical radial basis functions accompanied with the Chebyshev node generation is investigated for the solution of boundary value problems. In contrast to the traditional conical radial basis function method, where the collocation points are placed uniformly or quasi-uniformly in the physical domain of the boundary value problems in question, we consider three different Chebyshev-type schemes to generate the collocation points. This simple scheme improves accuracy of the method with no additional computational cost. Several numerical experiments are given to show the validity of the newly proposed method.
\end{abstract}

\section{Introduction}

The radial basis function- (RBF-) based meshless methods perform well for interpolating multidimensional scattered data during the past several decades $[1,2]$. These methods have been successfully used in a variety of applications owing to its spectral accuracy and simplicity [3-5].

Particularly, the Multiquadrics (MQ), which is also known as the Kansa method [6], is a widely used RBF. For traditional investigations, the source and collocation points are taken to be the same. Recently, Chen et al. [7, 8] proposed a novel approach to improve the performance of the Kansa method, where the source and collocation points are separated with the source points extended to a larger domain. This idea is analogous to the use of fictitious source points used in the method of fundamental solutions [9].

It should be noted that the shape parameter in the MQ plays an essential role for the numerical solution in terms of the accuracy and stability $[10,11]$. Thus, the selection of the shape parameter in MQ is a big issue and such a problem also occurs for some other radial basis functions such as the Gaussian radial basis function $[12,13]$. In this paper, our investigation focuses on the conical radial basis function which is a parameter-free function [14]. Motivated by the previous literature, we propose a new approach under Chebyshev-type schemes to improve the performance of the conical radial basis functions. This is fulfilled by coupling node generation under the Chebyshev-type schemes and the conical radial basis functions. In the newly proposed approach, no additional computational cost and fictitious points are needed while the solution accuracy is improved with easy implement. The proposed approach is also promising in dealing with fractional problems [15-18].

The structure of this paper is organized as below. In Section 2, we introduce the basic theory of the conical radial basis functions method. In Section 3, we propose three new approaches by placing the collocation points under the Chebyshev-type schemes on the physical domain. Followed by Section 4, the numerical implementation is given. Several numerical results, compared to the traditional conical radial basis function method, are presented in Section 5. Finally, some concluding remarks as well as ideas for future work are provided in Section 6 . 
TABLE 1: Example 1 Relative average errors for the traditional CRBF, CGR-RBF, CG-RBF, and CGL-RBF.

\begin{tabular}{lcccc}
\hline \multirow{2}{*}{ CRBF } & CGR-RBF & CG-RBF & CGL-RBF \\
& 441 & 437 & 437 & 437 \\
\hline$m=5$ & $2.05 \times 10^{-4}$ & $3.71 \times 10^{-6}$ & $2.42 \times 10^{-6}$ & $3.56 \times 10^{-6}$ \\
$m=7$ & $1.24 \times 10^{-5}$ & $1.28 \times 10^{-7}$ & $1.20 \times 10^{-7}$ & $1.09 \times 10^{-7}$ \\
$m=9$ & $9.23 \times 10^{-7}$ & $2.45 \times 10^{-8}$ & $1.07 \times 10^{-8}$ & $8.95 \times 10^{-9}$ \\
$m=11$ & $1.84 \times 10^{-7}$ & $2.00 \times 10^{-8}$ & $5.79 \times 10^{-8}$ & $1.21 \times 10^{-8}$ \\
\hline
\end{tabular}

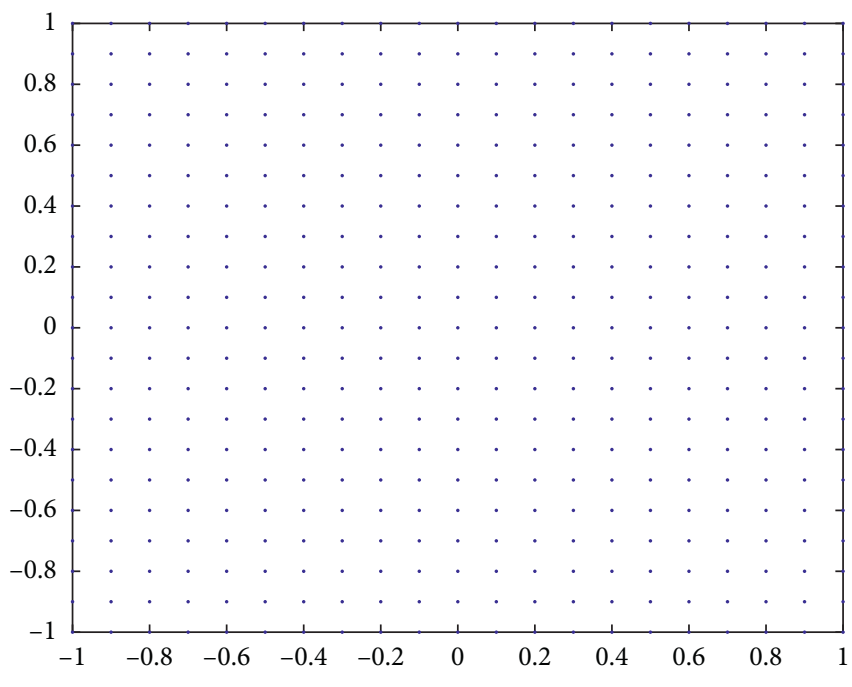

(a)

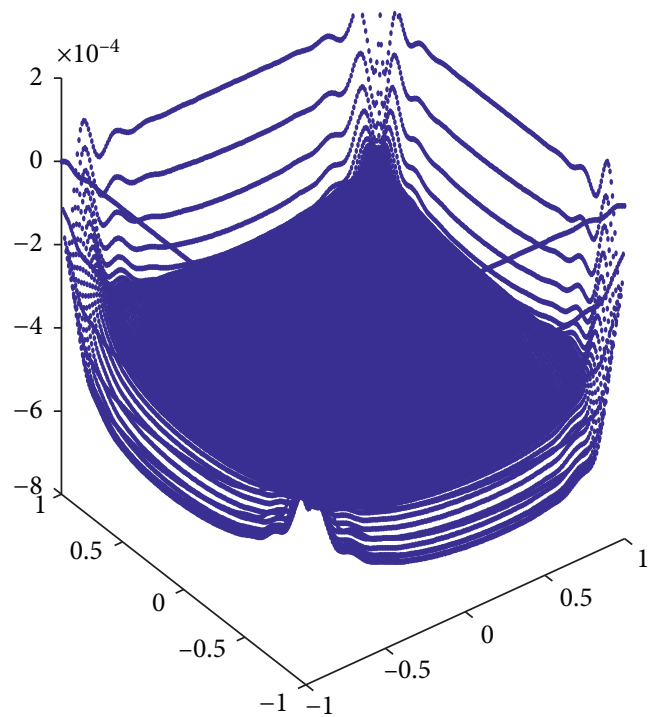

(b)

FIgURE 1: Configuration of the source points (a) and the corresponding absolute errors (b) for the traditional CRBF.

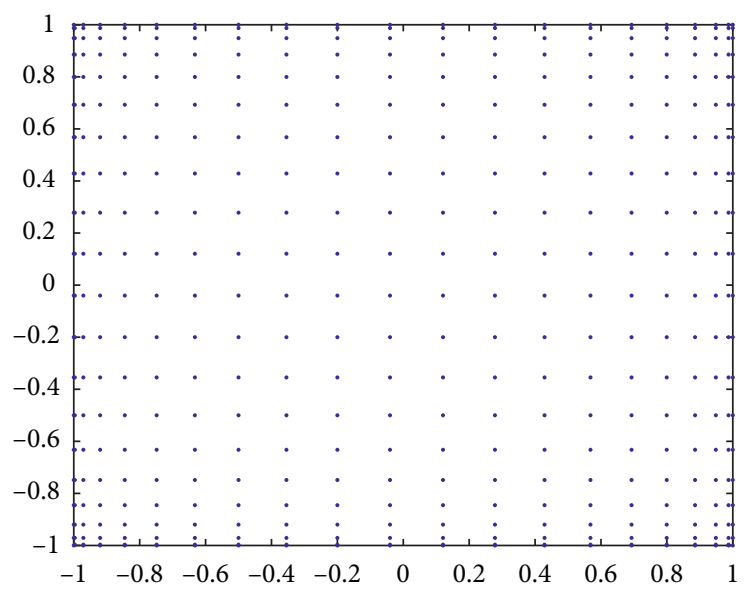

(a)

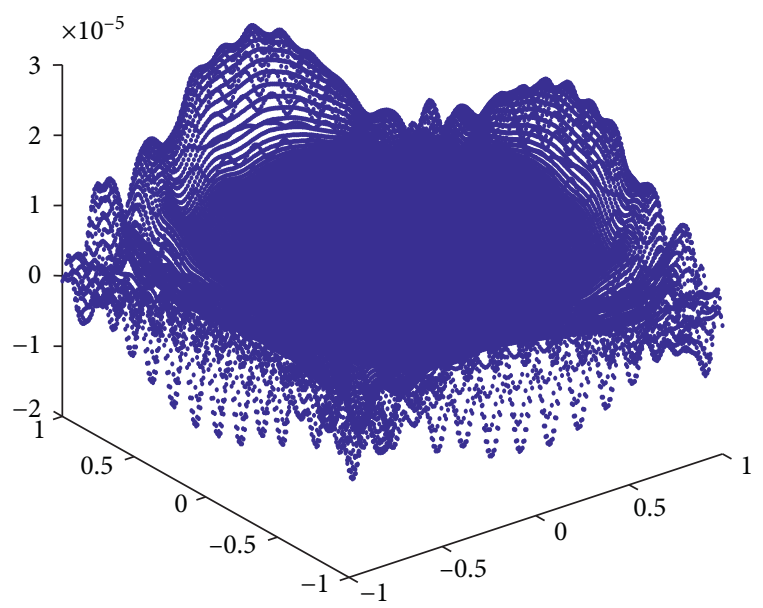

(b)

FIGURE 2: Configuration of the source points (a) and the corresponding absolute errors (b) for the CGR-RBF. 


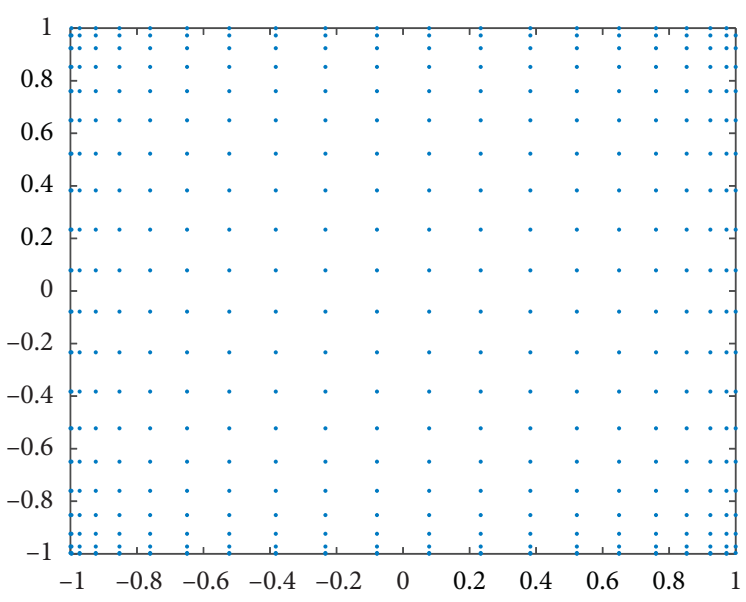

(a)

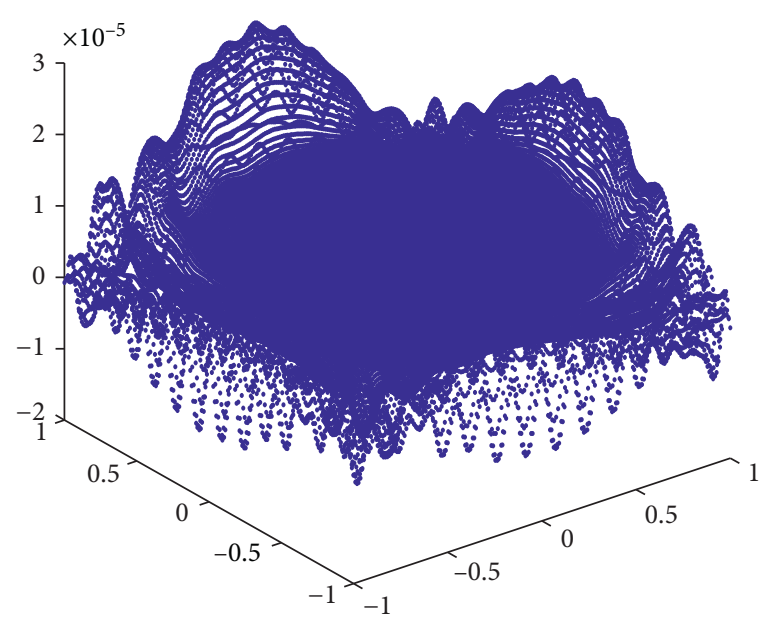

(b)

FIGURE 3: Configuration of the source points (a) and the corresponding absolute errors (b) for the CG-RBF.

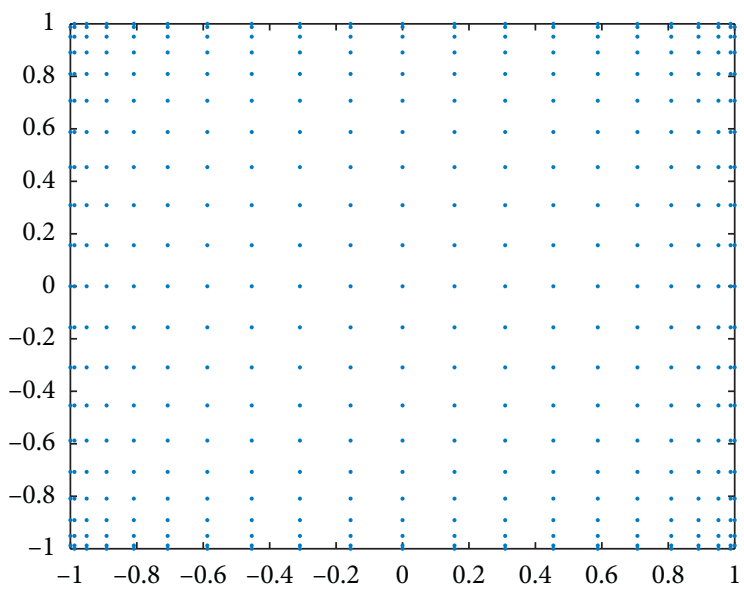

(a)

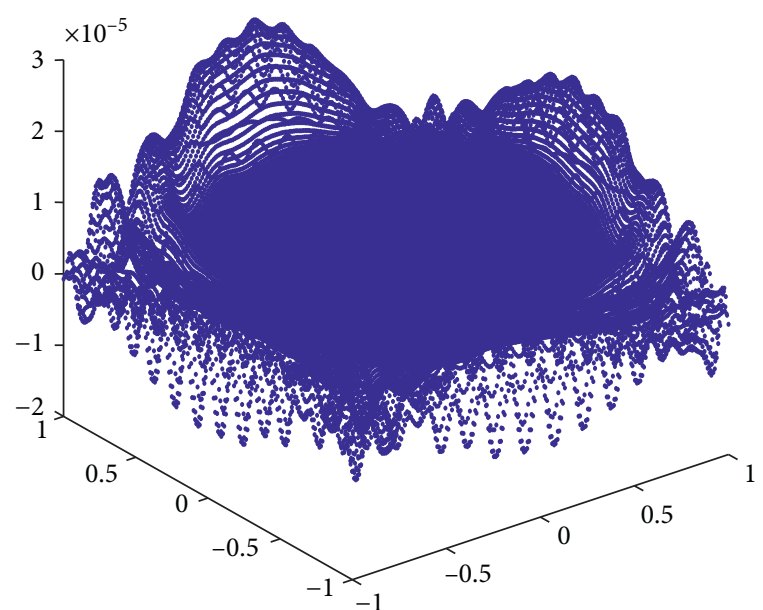

(b)

FIgure 4: Configuration of the source points (a) and the corresponding absolute errors (b) for the CGL-RBF.

\section{The Conical Radial Basis Functions (CRBF) Method}

For brevity, we consider the boundary value problems (BVPs) for elliptic partial differential equation (PDE) of second order:

$$
\begin{gathered}
a(P) \frac{\partial^{2} u}{\partial x^{2}}+b(P) \frac{\partial^{2} u}{\partial y^{2}}+c(P) \frac{\partial u}{\partial x}+d(P) \frac{\partial u}{\partial y} \\
+e(P) u=f(P), \quad P=(x, y) \in \Omega, \\
u(P)=\bar{u}(P), \quad P=(x, y) \in \Gamma_{D}, \\
\frac{\partial u(P)}{\partial n}=\bar{q}(P), \quad P=(x, y) \in \Gamma_{N},
\end{gathered}
$$

where $a(P), b(P), c(P), d(P)$, and $e(P)$ are coefficients of PDE, $\Omega \subset R^{2}$ is a two-dimensional physical domain, $\bar{u}(P)$ is the known boundary data on the Dirichlet boundary $\Gamma_{D}$, and $\bar{q}(P)$ is the known boundary data on the Neumann boundary $\Gamma_{N}$, with $\Gamma_{D} \cup \Gamma_{N}=\partial \Omega$ and $\Gamma_{D} \cap \Gamma_{N}=\phi$.

The basic theory of the CRBF method lies in the numerical solution of the BVP (1-3) being given by the following general formulation:

$$
\widetilde{u}(P)=\sum_{j=1}^{M} \alpha_{j} \varphi\left(\left\|P-P_{j}\right\|_{2}\right),
$$

where $M$ is the total number of source points $\left\{P_{j}\right\}_{j=1}^{M}$ on the whole physical domain $\bar{\Omega}=\Omega \cup \partial \Omega,\left\{\alpha_{j}\right\}_{j=1}^{M}$ are the required coefficients, $\varphi\left(\left\|P-P_{j}\right\|_{2}\right)=r_{j}^{m}$ is the CRBF , $m$ is a positive odd integer in the $\mathrm{CRBF}$, and 


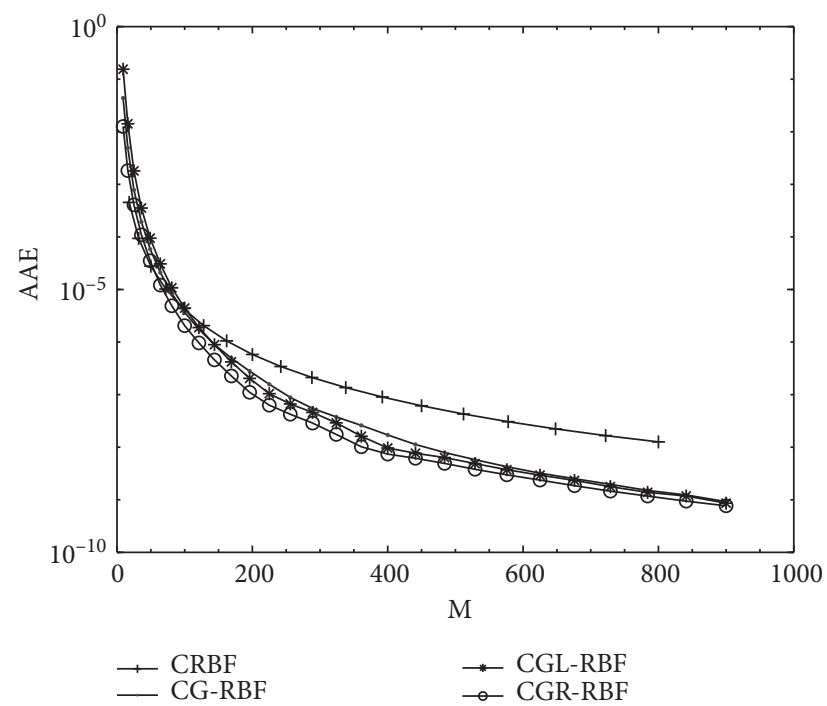

FIgURE 5: Configuration of the average absolute errors (AAE) for the traditional CRBF and the other three new schemes with fixed $m=5$.

TABLE 2: Example 2: relative average errors for the traditional CRBF, CGR-RBF, CG-RBF, and CGL-RBF.

\begin{tabular}{lcccc}
\hline \multirow{2}{*}{ CRBF } & CGR-RBF & CG-RBF & CGL-RBF \\
& 344 & 361 & 848 & 340 \\
\hline$m=3$ & $1.59 \times 10^{-5}$ & $8.67 \times 10^{-6}$ & $1.89 \times 10^{-6}$ & $8.49 \times 10^{-6}$ \\
$m=5$ & $1.30 \times 10^{-6}$ & $3.15 \times 10^{-8}$ & $2.12 \times 10^{-8}$ & $1.14 \times 10^{-7}$ \\
$m=7$ & $2.45 \times 10^{-7}$ & $1.62 \times 10^{-6}$ & $4.56 \times 10^{-9}$ & $2.31 \times 10^{-8}$ \\
$m=9$ & $9.23 \times 10^{-8}$ & $1.77 \times 10^{-6}$ & $9.36 \times 10^{-9}$ & $2.55 \times 10^{-9}$ \\
$m=11$ & $6.17 \times 10^{-8}$ & $4.09 \times 10^{-7}$ & & $2.52 \times 10^{-9}$ \\
\hline
\end{tabular}

$r_{j}=\left\|P-P_{j}\right\|=\sqrt{\left(x-x_{j}\right)^{2}+\left(y-y_{j}\right)^{2}}$ is the Euclidean norm distance between points $P=(x, y)$ and $P_{j}=\left(x_{j}, y_{j}\right)$.

We denote $\left\{P_{j}\right\}_{j=1}^{M_{I}}$ the collocation points inside the domain $\Omega,\left\{P_{j}\right\}_{j=1}^{M_{D} j}$ the collocation points on the Dirichlet boundary $\Gamma_{D}$, and $\left\{P_{j}\right\}_{j=1}^{M_{N}}$ the collocation points on the Neumann boundary $\Gamma_{N}$ with the total collocation number $M=M_{I}+M_{D}+M_{N}$.

The source points and the collocation points, usually uniformly located in the physical domain, are taken to be the same in the traditional RBF approach. Recently, Chen et al. $[6,7]$ proposed a simple approach for the Kansa method, where the source points can be located outside it. The ghost (fictitious) circle in 2D and a sphere (or an ellipsoid) in $3 \mathrm{D}$ are considered. In this paper, we consider the source points generation in a new way.

\section{The Chebyshev-Type Schemes}

The main idea is to use the Chebyshev-type schemes, which is generated in the interval $(-1,1)$, instead of the traditional uniformly distributed source points. It should be noted that the Chebyshev collocation method is usually used to find the approximate solutions of differential equations using a truncated Chebyshev series $[19,20]$. The novelty of the idea in this paper lies in that the points generated by the Chebyshev-type schemes are combined with the CRBF, while the computational cost remains the same as the traditional way and there is no need to consider the fictitious points. $\mathrm{Nu}$ merical results in the numerical section will show the performance of the new way. To the best of our knowledge, this is the first time that such a technique is proposed in RBF collocation methods. The definite generations on each direction of the physical domains for three Chebyshev-type nodes are shown below.

Scheme 1 (Chebyshev-Gauss: CG)

$$
P_{j}=\cos \frac{(2 j+1) \pi}{2 n+2}, \quad 0 \leq j \leq n
$$

Note that, for arbitrary interval $[a, b]$, an affine transformation can be used:

$$
P_{j}=\frac{1}{2}(a+b)+\frac{1}{2}(b-a) \cos \frac{(2 j+1) \pi}{2 n+2}, \quad 1 \leq j \leq n .
$$

This transformation can be easily extended to the following two schemes.

Scheme 2 (Chebyshev-Gauss-Radau: CGR)

$$
\begin{aligned}
& P_{0}=1, \\
& P_{j}=\cos \frac{2 \pi j}{2 n+1}, \quad 1 \leq j \leq n .
\end{aligned}
$$


Scheme 3 (Chebyshev-Gauss-Lobatto: CGL)

$$
P_{j}=\cos \frac{\pi j}{n}, \quad 1 \leq j \leq n-1 \text {. }
$$

The main differences among these three newly proposed schemes lie in the position of the collocation points. The figures of the three schemes will be shown in the following numerical section to verify the performance of the proposed schemes.

\section{Numerical Implementation}

In the numerical implementation of the RBF, equation (4) is forced to satisfy equations (1)-(3) at all collocation points which is the same as source points $\left\{P_{j}\right\}_{j=1}^{M}$, and we have

$$
\begin{gathered}
\sum_{i=1}^{M} \alpha_{i} L \varphi\left(P_{i}, P_{j}\right)=f\left(P_{i}\right), \\
P_{j}=\left(x_{j}, y_{j}\right) \in \Omega, \quad j=1, \ldots, M_{I}, \\
\sum_{i=1}^{M} \alpha_{i} \varphi\left(P_{i}, P_{j}\right)=\bar{u}\left(P_{i}\right), P_{j}=\left(x_{j}, y_{j}\right) \in \Gamma_{D}, \\
j=M_{I}+1, \ldots, M_{I}+M_{D}, \\
\sum_{i=1}^{M} \alpha_{i} \frac{\partial \phi\left(P_{i}, P_{j}\right)}{\partial n}=\bar{q}\left(P_{i}\right), P_{j}=\left(x_{j}, y_{j}\right) \in \Gamma_{N}, \\
j=M_{I}+M_{D}+1, \ldots, M,
\end{gathered}
$$

where

$$
L \varphi=\left(a(P) \frac{\partial^{2}}{\partial x^{2}}+b(P) \frac{\partial^{2}}{\partial y^{2}}+c(P) \frac{\partial}{\partial x}+d(P) \frac{\partial}{\partial y}+e(P)\right) \varphi
$$

Equations (9)-(11) yield the $M \times M$ system:

$$
Q \alpha=b,
$$

where

$$
\begin{aligned}
Q_{i j}= & \left(a\left(P_{j}\right) \frac{\partial^{2}}{\partial x^{2}}+b\left(P_{j}\right) \frac{\partial^{2}}{\partial y^{2}}+c\left(P_{j}\right) \frac{\partial}{\partial x}+d\left(P_{j}\right) \frac{\partial}{\partial y}\right. \\
& \left.+e\left(P_{j}\right)\right) \varphi\left(P_{i}, P_{j}\right), \quad j=1, \ldots, M_{I}, \\
Q_{i j}= & \varphi\left(P_{i}, P_{j}\right), \quad j=M_{I}+1, \ldots, M_{I}+M_{D}, \\
Q_{i j}= & \frac{\partial \varphi\left(P_{i}, P_{j}\right)}{\partial n}, \quad j=M_{I}+M_{D}+1, \ldots, M, \\
b= & \left(f_{1}, \ldots, f_{M}, \bar{u}_{M+1}, \ldots, \bar{u}_{N_{D}}, \bar{q}_{N_{D}+1}, \ldots, \bar{q}_{N}\right)^{T},
\end{aligned}
$$

$\alpha=\left(\alpha_{1}, \alpha_{2}, \ldots, \alpha_{N}\right)^{T}$ and $(\cdot)^{T}$ is the transpose of vector.

This procedure is the same for the traditional source points and the source points generated by the Chebyshevtype schemes (5)-(8).

\section{Numerical Results}

To measure the accuracy, we compute the relative average errors (RAE) of the exact and approximate solutions for all cases.

Example 1. We take the exact solution of (1)-(3) as

$$
u(x, y)=\left(x-x^{2}\right)\left(y-y^{2}\right)
$$

and the domain $\Omega=[-1,1] \times[-1,1]$ with the Dirichlet $\bar{u}(x, y)=u(x, y)$ and Neumann data $\bar{q}(x, y)=\partial u / \partial n$ on the boundary, and the coefficients of (1)-(3) are

$$
\begin{aligned}
& a(x, y)=2-x^{2}-y^{2}, \\
& b(x, y)=e^{x-y} \\
& c(x, y)=-2 x \\
& d(x, y)=-e^{x-y} \\
& e(x, y)=0 \\
& f(x, y)=\operatorname{Lu}(x, y) .
\end{aligned}
$$

Here, the total collocation point number of the traditional CRBF is $M=441$, since the points generated by the Chebyshev-type schemes are not uniformly chosen, the corresponding total collocation point numbers of the CGR, CG, and CGL are the same, i.e., $M=437$. The computational point number is the same, i.e., $N_{t}=40401$.

In this example, there is singularity at origin for $m=3$, so we use $m=5, m=7, m=9$, and $m=11$ in the CRBF $\varphi(\cdot)=r^{m}$. The lists of relative average errors are shown in Table 1. It is clear that the numerical solution accuracy increases with the increasing number $m$. For larger $m(m \geq 13)$, the solution accuracy almost remains the same. This is eliminated here and thereafter. Besides, we can see that the relative average errors of the CGR-RBF, CG-RBF, and CGL-RBF are superior than the tradition CRBF case with two decimals for $m=5$ and $m=7$. For $m=9$, the relative average errors of the CGR-RBF and CG-RBF are more accurate than the CRBF case with one decimal and the CGL-RBF are more accurate than the CRBF case with two decimals.

More specifically, the configuration of the source points and the corresponding absolute errors for the traditional CRBF and the other three new schemes for $m=5$ are shown in Figures 1-4. It is shown that the main differences among the traditional points and the points generated by three newly proposed schemes lie in the position. The points generated by three newly proposed schemes cluster at the corner points and scatter at center points, while the traditional points are uniformly distributed in the whole physical domain.

We find that the absolute errors shown in Figure 1 are relatively large at corner points. However, for the three newly proposed schemes shown in Figures 2-4, the absolute errors at the corner points are small. This phenomenon reveals that the three newly proposed schemes can improve the solution accuracy at corner points. 
To investigate the stability of the method, the configuration of the average absolute errors (AAE) for the traditional CRBF and the other three new schemes with fixed $m=5$ are shown in Figure 5, from which we can find that all methods are stable. For small collocation point numbers $(M<200)$, all methods have the same solution accuracy, but for large collocation point numbers, the three newly proposed schemes perform better than the traditional CRBF.

Example 2. We take the exact solution of (1)-(3) as

$$
u(x, y)=-\frac{1}{2} x+y+\frac{1}{4}\left(x^{2}-y^{2}\right)
$$

and the connected domain $\Omega=\Omega_{2}-\Omega_{1}$ with outer boundary $\Omega_{2}=[-2,2] \times[-2,2]$ and inner boundary $\Omega_{1}=[-1,1] \times[-1,1]$, and only the Dirichlet boundary $\bar{u}(x, y)=u(x, y)$ is considered, and the coefficients of (1) $-(3)$ are

$$
\begin{aligned}
& a(x, y)=b(x, y)=1, \\
& c(x, y)=d(x, y)=e(x, y)=0, \\
& f(x, y)=\operatorname{Lu}(x, y)
\end{aligned}
$$

In this example, the total collocation point numbers are $M=344$ for the traditional $\mathrm{CT}, M=361$ for the CGR, $M=348$ for the CG, and $M=340$ for the CGL. The computational point number is $N_{t}=1028$ for this example. The lists of relative average errors are shown in Table 2. For all numbers of $m$, numerical results show that the relative average errors of the CG-RBF and CGL$\mathrm{RBF}$ are more accurate than the tradition CRBF case with one decimal. For the CGR-RBF, the solution accuracy is not stable. This scheme should be carefully chosen in future applications. We suggest using the CG$\mathrm{RBF}$ and the CGL-RBF schemes in relative investigations.

\section{Conclusions}

In this paper, we investigate the parameter-free conical radial basis function. A novel collocation technique is proposed which improves the performance of the traditional conical radial basis function method by utilizing Chebyshev-type schemes. Our results show that the most attractive features of the proposed method are its simplicity and the corresponding numerical solution accuracy. The method can be extended directly to threedimensional problems and will be applied to other challenging problems in the future.

\section{Data Availability}

The data used to support the findings of this study are available from the corresponding author upon request.

\section{Conflicts of Interest}

The authors declare that there are no conflicts of interest regarding the publication of this paper.

\section{Acknowledgments}

The work was supported by the Natural Science Foundation of Anhui Province (Project no. 1908085QA09) and University Natural Science Research Project of Anhui Province (Project no. KJ2019A0591, KJ2017B015, and KJ2020B06).

\section{References}

[1] B. Fornberg and N. Flyer, "Solving PDEs with radial basis functions," Acta Numerica, vol. 24, pp. 215-258, 2015.

[2] A. I. Rozhenko, "Comparison of radial basis functions," Journal of the Society for Industrial and Applied Mathematics, vol. 21, pp. 273-290, 2018.

[3] X. Song, Z. Y. Bi, S. X. Jiang, Y. X. Jin, and M. S. Yang, “Thin plate spline radial basis function for the free vibration analysis of laminated composite shells," Composite Structures, vol. 93, pp. 611-615, 2011.

[4] Y.-F. Zhang and C.-J. Li, "A Gaussian RBFs method with regularization for the numerical solution of inverse heat conduction problems," Inverse Problems in Science and Engineering, vol. 24, no. 9, pp. 1606-1646, 2016.

[5] D. Sharanjeet, A. Turgut, and A. Gokhan, "Algorithms for numerical solution of the equal width wave equation using multi-quadric quasi-interpolation method," International Journal of Modern Physics C, vol. 30, p. 1950087, 2019.

[6] E. J. Kansa, "Multiquadrics-a scattered data approximation scheme with applications to computational fluid-dynamics-II solutions to parabolic, hyperbolic and elliptic partial differential equations," Computers \& Mathematics with Applications, vol. 19, no. 8-9, pp. 147-161, 1990.

[7] C. S. Chen, A. Karageorghis, and F. Dou, "A novel RBF collocation method using fictitious centres," Applied Mathematics Letters, vol. 101, p. 106069, 2020.

[8] J. Lin, Y. X. Zhao, D. Watson, and C. S. Chen, "The radial basis function differential quadrature method with ghost points," Mathematics and Computers in Simulation, vol. 173, 2020.

[9] Z.-C. Li, Y. Wei, Y. Chen, and H.-T. Huang, "The method of fundamental solutions for the helmholtz equation," Applied Numerical Mathematics, vol. 135, pp. 510-536, 2019.

[10] G. E. Fasshauer and J. G. Zhang, "On choosing "optimal"shape parameters for RBF approximation," Numerical Algorithms, vol. 45, no. 1-4, pp. 345-368, 2007.

[11] W. Chen, Y. Hong, and J. Lin, "The sample solution approach for determination of the optimal shape parameter in the Multiquadric function of the Kansa method," Computers \& Mathematics with Applications, vol. 75, no. 8, pp. 2942-2954, 2018.

[12] L.-T. Luh, "The shape parameter in the Gaussian function II," Engineering Analysis with Boundary Elements, vol. 37, no. 6, pp. 988-993, 2013.

[13] A. R. Lamichhane and C. S. Chen, "The closed-form particular solutions for Laplace and biharmonic operators using a Gaussian function," Applied Mathematics Letters, vol. 46, pp. 50-56, 2015.

[14] J. C. Li, "A radial basis meshless method for solving inverse boundary value problems," Int. J. Numer. Meth. Bio.vol. 20, pp. 51-60, 2004.

[15] I. Ahmad, S. Islam, M. Siraj-ul-Islam, S. Mehnaz, and S. Zaman, "Local meshless differential quadrature collocation method for time-fractional PDEs," Discrete \& Continuous Dynamical Systems - S, vol. 13, no. 10, p. 2641, 2020.

[16] I. Ahmad, H. Ahmad, M. Inc, S. W. Yao, and B. Almohsen, "Application of local meshless method for the solution of two 
term time fractional-order multi-dimensional PDE arising in heat and mass transfer," Thermal Science, vol. 24, no. s1, pp. S95-S105, 2020.

[17] M. Inc, M. N. Khan, I. Ahmad, S.-W. Yao, H. Ahmad, and P. Thounthong, "Analysing time-fractional exotic options via efficient local meshless method," Results in Physics, vol. 19, Article ID 103385, 2020.

[18] I. Ahmad, H. Ahmad, P. Thounthong, Y. Chu, and C. Clemente, "Solution of multi-term time-fractional pde models arising in mathematical biology and physics by local meshless method," Symmetry, vol. 17, no. 7, p. 1195, 2020.

[19] A. Akyüz and M. Sezer, "A Chebyshev collocation method for the solution of linear integro-differential equations," International Journal of Computer Mathematics, vol. 72, no. 4, pp. 491-507, 1999.

[20] G. Yuksel, O. R. Isik, and M. Sezer, "Error analysis of the Chebyshev collocation method for linear second-order partial differential equations," International Journal of Computer Mathematics, vol. 92, no. 10, pp. 2121-2138, 2015. 